\title{
Comentarios acerca de la carta el editor Estado nutricional en una población pediátrica de una zona rural de Cusco, Perú
}

\author{
Comments about letter Nutritional status in a pediatric population in a rural area from Cusco, Perú
}

\section{Sr Editor:}

Al revisar la publicacion de su revista, en la que se describe el estado nutricional de una poblacion infantil rural (1), cabe hacer algunas observaciones al artículo en mención:

Son relevantes los estudios que acercan a los estudiantes hacia la realidad local, describiendola y dando a conocer los hallazgos; este aspecto es un buen aporte de la publicación.

Los paises en vias de desarrollo y en transición epidemiológica muestran avances con respecto a los países de bajos ingresos en términos de desnutrición. El estado nutricional no se puede restringir a la valoracion de peso, talla e indice de masa corporal (IMC) como se señala en el articulo, sino que es un constructo mas amplio, donde se valora la historia clinica, las practicas de alimentación, adecuado consumo de micronutrientes, estado de hierro, comorbilidades, actividad fisica entre otros. Hacer uso de peso, talla e IMC debe llamarse mas propiamente antropometria (2).

Conocer el número de niños excluídos del estudio en virtud a sus antecedentes clínicos sería útil además de los 368 descritos.

Por otro lado, se observa que en la publicación se hace uso de las tablas de la Organización Mundial de la Salud (OMS), publicadas en el año 2006, para comparar los estandares de antropometria; sin embargo, se hace referencia a terminos como desnutricion cronica, desnutricion aguda y desnutricion global, los mismos que no se encuentran en el módulo de interpretacion de los estandares de la OMS del año 2008 (3). Si se toma las categorías de desnutrición aguda, crónica y global como no mutuamente excluyentes hay niños que pueden estar en mas de un categoría y modificar el porcentaje de esta.

En la tabla 1 del mencionado trabajo (1), se hace evaluación de los 3 parametros habituales para los niños menores de 5 años, catalogando como mala nutricion al $69,7 \%$ de los niños estudiados, mientras en el grupo de 5 a 11 años, solo se usa el referente de IMC, sin evaluar la estatura para la edad que podria hablar de talla corta referencial. No se señala en la publicacion la razon por la que no se uso el IMC despues de los 3 años, según lo sugerido por la OMS.

Se señala una mejor condicion de nutricion en el grupo de escolares, asumiendo la intervencion de programas de alimentacion complementaria; esto no es optimo ya que se ha comparado ambos grupos con parametros diferentes y el diseño de estudio no permite atribuir ni explorar causalidad. Por otro lado, los efectos mas criticos de la alimentacion se dan en los primeros dos años de vida (4), de esta manera cualquier intervencion despues de este periodo tiene menor impacto en el desarrollo del niño y la futura vida del adulto (5).

\section{Ronny Breibat-Timpo ${ }^{1, a}$}




\section{Correspondencia:}

Ronny Breibat Timpo

Urbanizacion Progreso I-10, Cusco, Perú.

Correo electrónico: breibatman@hotmail.com

Telefono: 51984392426

\section{REFERENCIAS BIBLIOGRÁFICAS}

1. Monterroso-Cusihuallpa C, Rondon-Abuhadba EA, Atamari-Anahui N, Tupayachi-Atapaucar ME. Estado nutricional en una población pediátrica de una zona rural de Cusco, Perú. Rev Med Hered. 2018; 29:203204.

2. Wiskin A, Johnson M, Leaf A, Wootton S, Beattie M. How to use: nutritional assesment in children. Arch Dis Child Educ Pract Ed. 2015; 100:204-9.

3. World Hearth Organization. Training Course on Child Growth Assesment. Geneva: World Hearth Organization; 2018.
4. Schwarzenberg S, Georgieff M. Advocacy for improving nutrition in the first 1000 days to support chilhood development and adult health. Pediatrics. 2018; 141(2):0-0. doi: 10.1542/peds.2017-3716

5. Fewtrell M, Bronsky J, Campoy C, et al. Complementary feeding: A position paper by the European Society for Paediatric Gastroenterology, Hepatology, and Nutrition (ESPGHAN) Committe on Nutrition. J Pediatr Gastroenterol Nutr. 2017; 64(1):119-132. doi: 10.1097/MPG.000000000000 1454

Recibido: $12 / 11 / 2018$ 